\section{Integralidade da atenção e integração de serviços de saúde: desafios para avaliar a implantação de um "sistema sem muros"}

\author{
Comprehensive health care and integrated \\ health services: challenges for evaluating \\ the implementation of a "system without walls"
}

Zulmira M. de Araújo Hartz 1,2 André-Pierre Contandriopoulos 2

\footnotetext{
1 Escola Nacional de Saúde Pública, Fundação Oswaldo Cruz, Rio de Janeiro, Brasil.

2 Faculté de Médecine, Université de Montréal, Montréal, Canada.

Correspondência Z. M. A. Hartz Départment de Médecine Sociale et Préventive, Université de Montréal. 2375 Côte Sainte Catherine \# 6046, Montréal, H36 $3 J 7$ Qc, Canada. zulmira.hartz@umontreal.ca
}

\begin{abstract}
The premise of this paper is that comprehensive health care is a major component in the investigation and evaluation of health services and systems, structured as inter-organizational health care networks articulating clinical, functional, normative, and systemic dimensions in their operationalization and based on the understanding that no organization combines all the necessary resources and capabilities to solve the health problems of a population with its various life cycles. Given the complex nature of this "system without walls", eliminating barriers to access in the various health care levels in response to local and regional health, we take this opportunity to share a few "preliminary lessons" from our experience and from the literature on integrated health services which may interest researchers and managers concerned with the implementation of such services.
\end{abstract}

Health Services; Services Evaluation; Systems Integration

\section{Introdução}

Em face do problema da fragmentação dos modelos assistenciais, a integração dos serviços de saúde aparece como atributo inerente às reformas das políticas públicas fundamentadas na Atenção Primária da Saúde (APS). Teoricamente, integração significa coordenação e cooperação entre provedores dos serviços assistenciais para a criação de um autêntico sistema de saúde, mas, na prática, isso ainda não se realizou e poucas são as iniciativas para o monitoramento e avaliação sistemática de seus efeitos 1. No Brasil, a integralidade da atenção é um dos seus pilares de construção, baseada na primazia das ações de promoção; garantia de atenção nos três níveis de complexidade; articulação das ações de prevenção, promoção e recuperação; abordagem integral dos indivíduos e das famílias 2 .

$\mathrm{O}$ conceito de integralidade remete, portanto, obrigatoriamente, ao de integração de serviços por meio de redes assistenciais, reconhecendo a interdependência dos atores e organizações, em face da constatação de que nenhuma delas dispõe da totalidade dos recursos e competências necessários para a solução dos problemas de saúde de uma população em seus diversos ciclos de vida. Torna-se, portanto, indispensável desenvolver mecanismos de cooperação e coordenação próprios de uma gestão eficiente e responsável dos recursos co- 
letivos, a qual responda às necessidades de saúde individuais em âmbitos local e regional.

A concretização dessa imagem ideal de um "sistema sem muros", eliminando as barreiras de acesso entre os diversos níveis de atenção do domicílio ao hospital, especialistas e generalistas, setores público e privado -, ligados por corredores virtuais que ultrapassem de fato as fronteiras municipais com vistas à otimização de recursos e ampliação das oportunidades de cuidados adequados, embora consensual em seus princípios, tem se mostrado de difícil realização, demandando estudos de avaliação que contribuam para a sua efetiva implantação.

Este texto tem, portanto, o objetivo de compartilhar lições preliminares destacadas em algumas experiências sobre a avaliação da integração de serviços que julgamos de interesse comum a gestores e avaliadores de redes assistenciais. A exposição se organiza em dois eixos: uma síntese conceitual sobre as diversas linguagens e dimensões da integração de serviços no campo da saúde, seguida de algumas diretrizes práticas a serem consideradas na pesquisa avaliativa sobre sua implantação e efetividade.

\section{O que entendemos por sistemas integrados de saúde ou redes assistenciais?}

Num sentido mais amplo, a integração em saúde é um processo que consiste em criar e manter uma governança comum de atores e organizações autônomas, com o propósito de coordenar sua interdependência, permitindo-lhes cooperar para a realização de um projeto (clínico) coletivo ${ }^{3}$. Essa perspectiva de sistema integrado de saúde tem como representação nuclear uma rede de cuidados com múltiplas dimensões de integração entre os diferentes subsistemas relacionando a Clínica e a Governança às representações e valores coletivos (Figura 1).

A integração propriamente dita dos cuidados consiste em uma coordenação durável das práticas clínicas destinadas a alguém que sofre com problemas de saúde, visando a assegurar a continuidade e a globalidade dos serviços requeridos de diferentes profissionais e organizações, articuladas no tempo e no espaço, conforme os conhecimentos disponíveis. A integração das equipes clínicas (alguns autores referindo-se apenas à equipe médica) tem como principais atributos a constituição multidisciplinar de seus integrantes e sua inserção estrutural e participativa na rede dos cuidados. Para efeitos práticos, ela pode ser agrupada sob a denominação integração clínica, a qual se apóia em estratégias de gestão, do financiamento e da informação (integração funcional), que assegurem uma coordenação comum, orientada por um sistema de informações ágil e flexível, capaz de tomar decisões sobre o compartilhamento de responsabilidades, atribuições e recursos financeiros.

Essas dimensões da integração corresponderiam, na tipologia proposta por Mendes, à gestão da clínica e à dos pontos de atenção em que a oferta e a utilização de serviços são feitas pelo "conjunto dos pontos de atenção à saúde que se articulam, pela via das tecnologias de gestão da clínica, numa rede capaz de prestar atenção contínua a uma população adscrita" (Mendes EV, comunicação pessoal, 2001). Para o autor, o quase desconhecimento dessas tecnologias de microgestão - gestão da utilização de procedimentos, perfilização clínica, gestão da patologia, gestão de casos, lista de espera e procedimentos expectantes, revisão do usuário, protocolos clínicos e sistema integrado de informação - estaria dificultando a implantação nacional dos sistemas de referência e contra-referência dos pacientes, apesar de freqüentemente enunciados.

É importante conceituar ainda as duas outras dimensões - integração normativa e integração sistêmica - propostas por Contandriopoulos et al. 3 , particularmente em sistemas públicos de saúde como o do Canadá e o do Brasil. A integração normativa visa a garantir a coerência entre o sistema de representações e valores dos atores em simultaneidade com as interfaces da integração clínica/funcional. Já a integração sistêmica interessa-se pela coerência das diferentes modalidades integracionais em todos os níveis de atuação (organização, território, região, estados etc.), compreendendo que um projeto clínico que responda à complexidade e à incerteza dos problemas de saúde não pode resultar apenas das relações entre organizações e profissionais, as relações locais repercutindo nos demais níveis e instâncias políticas/decisórias.

\section{Que percursos priorizar na avaliação de uma rede dos serviços de saúde?}

Nas diversas reformas dos sistemas nacionais de saúde, a descentralização e a regionalização, mediante a integração dos serviços de saúde em redes assistenciais, têm sido ativamente estimuladas com base no "modelo ideal" de integração descrito, que teria como pressuposto teórico aumentar a efetividade e a eficiência dos sistemas. 


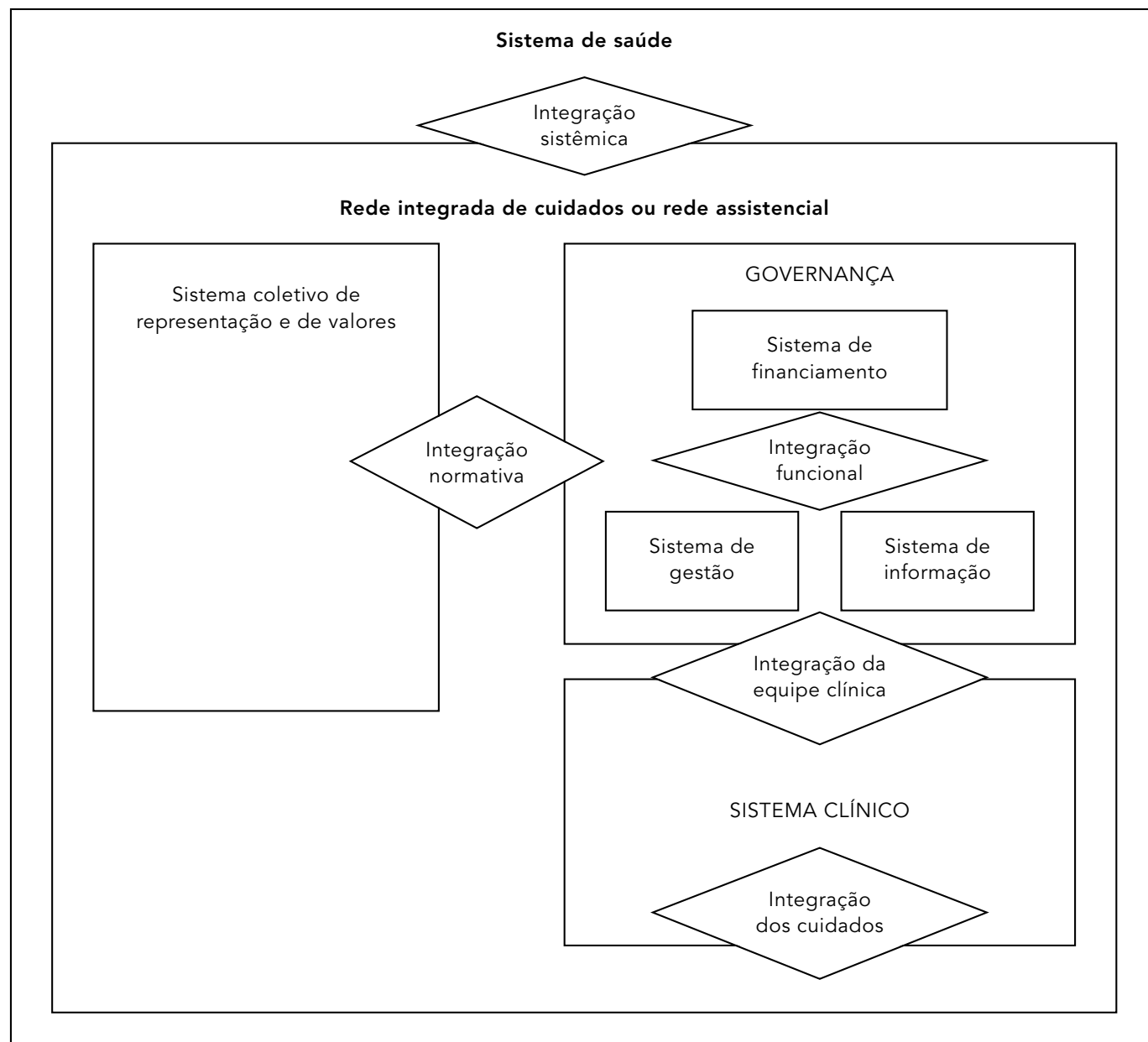

Tempo

Os discursos e as políticas sobre a integração fundamentam-se, assim, em três linguagens: a estrutural (modificação das fronteiras das organizações), a clínica (modificações nas práticas profissionais) e a da cooperação (novos formatos de negociação e de acordos entre atores e organizações). Em qualquer linguagem com que a integração se expresse, no entanto, a incerteza é permanente, uma vez que os diversos atores e organizações envolvidos não têm os mesmos recursos, interesses ou competências para se engajar em um projeto clínico nessa nova (e mais exigente) modalidade de cooperação, como vêm demonstrando alguns estudos canadenses 1,4,5,6.

A primeira questão a ser respondida relaciona-se, portanto, à própria implantação da rede, ao se indagar sobre a sua "real" existência em relação ao "modelo ideal". Esse questionamento deve ser feito antes que se indague sobre sua efetividade e eficiência, ou seja, se ela responde melhor aos objetivos de qualidade e de integralidade desejados, e a que custo, do que outras modalidades de intervenção. Essa abordagem coincide com as diretrizes para avaliação em redes, tomando-se por base a experiência de implementação das redes regionais da França 7,8,9,10. Como decorrência prática dessa constatação, a elaboração do modelo teórico idealizado, ou sua "imagem-objetivo", impõe-se como etapa indispensável à avaliabilidade do sistema em pauta. Na elaboração do modelo, os avaliadores devem levar em conta não apenas a documentação normativa e a re- 
visão da literatura disponível, mas também a percepção dos diferentes atores implicados, possibilitando estimar o "grau de implantação ou de fidelidade", mediante a utilização de indicadores compostos, escores ou "índice de integração”, as técnicas de consenso tendo sido fortemente recomendadas por alguns autores para tais fins $1,2,3,4,6,11,12$.

Os estudos de revisão da literatura mostram a produção de centenas de artigos voltados para doenças ou cuidados a grupos específicos (redes de atenção perinatal, AIDS, saúde mental etc.), mas são poucas as referências ao desempenho de sistemas no seu conjunto. $\mathrm{O}$ foco tem privilegiado a análise da coordenação em nível corporativo e hospitalar, com pouca ênfase na atenção ambulatorial ou domiciliar e na trajetória dos pacientes no continuum dos cuidados 1,9. Esta última lacuna pode ser superada utilizando-se os dez critérios propostos por Leatt et al. 1, para que os pacientes possam se assegurar de que realmente existe um sistema integrado de serviços:

- não precisar repetir sua história em cada atendimento;

- não precisar se submeter a repetições inúteis de exames;

- não ser a única fonte de informação para que o médico ou outro profissional conheça a sua história de utilização de serviços, procedimentos e medicamentos;

- não se restringir a um nível de atenção inadequado por incapacidade de atendimento em outro nível (superior ou inferior);

- dispor de 24 horas de acesso a um provedor de serviços ligado à atenção primária;

- receber informação clara e precisa sobre a qualidade da assistência e resultados esperados das opções terapêuticas, permitindo-lhe fazer escolhas esclarecidas;

- ter acesso fácil (por exemplo, telefone) e oportuno aos diversos exames e profissionais;

- dispor de tempo suficiente nas consultas do primeiro nível de atenção;

- ser rotineiramente contatado para prevenir complicações de problemas crônicos;

- receber apoio domiciliar e treinamento em autocuidado que maximize a autonomia.

No que concerne à lacuna da atenção ambulatorial e do enfoque sistêmico na produção do conhecimento científico, em virtude da variabilidade dos problemas de saúde contemplados, a utilização de medidas de integralidade relacionadas a "condições traçadoras" previamente estabelecidas facilita a definição de focos nas avaliações das ações programáticas de saúde, abrangendo os múltiplos "pontos de atenção” envolvidos na integração dos servi- ços. Já o uso de trajetória de pacientes, eventos-sentinela e monitoramento de hospitalizações evitáveis por uma atenção ambulatorial de qualidade é particularmente indicado para a detecção de passagens críticas no sistema, permitindo analisar suas causas e acompanhar o progresso de sua superação 10,12,13.

Finalizando esta sumarização sobre os aspectos referentes à avaliação da implantação dos sistemas integrados, não poderíamos deixar de retomar o questionamento de Leatt et al. 1: quem deveria realizar as atividades de avaliação? Nesse sentido, reforçaríamos a idéia (já experimentada pelos Estados Unidos e Inglaterra) da formação de um conselho ou comissão independente, com a presença de usuários e provedores, que controlasse a qualidade, os problemas e os resultados dos cuidados prestados, com os dados de monitoramento, contratando, quando pertinente, pesquisadores e consultores para as avaliações necessárias. Lembramos que a utilização de condições traçadoras, das demais ferramentas acima referidas e de outros dados dos sistemas de informação, poderia fazer parte tanto do sistema de monitoramento como dos estudos de avaliação, aumentando o potencial de utilidade dos resultados das pesquisas, como se verificou nos estudos de Campbell 14 e Brindis et al. 11 .

\section{Conclusões}

Sobre a natureza do objeto "rede", uma dupla leitura torna-se indispensável: como estrutura organizacional, voltada para a produção de serviços, e como uma dinâmica de atores em permanente renegociação de seus papéis, favorecendo novas soluções para velhos problemas num contexto de mudanças e compromissos mútuos.

Os argumentos também nos parecem suficientes para demonstrar a importância de que a intendência prevaleça sobre as intenções na formulação das políticas de integração, pois os méritos das inovações são muitas vezes sabotados pela usura de sua aplicação. Assim, são comuns situações em que rígidos mecanismos de controle e territorialização se antecipam à devida alocação de fundos e aos necessários investimentos relacionados aos aspectos do envolvimento cognitivo e afetivo dos profissionais implicados, quando é evidente a importância de incentivos à sua formação na constituição das redes assistenciais. Os estudos sobre a análise de implantação 15 podem contribuir, apontando precocemente essas contradições entre o "ideal e real" ao tratar "o como e o por- 
quê" do (in)sucesso das políticas de regionalização. Não se trata de adotar uma atitude de desconfiança relativa aos processos de implantação, e sim uma necessária precaução em face do reconhecimento de sua complexidade, de modo que a integridade das intervenções, nos diferentes contextos, não comprometa todo o esforço político institucional da integração de serviços que garantam a desejável integralidade da atenção em saúde.

No Brasil, apesar de ainda incipiente, a implantação de redes de atenção em saúde mental, saúde do idoso e saúde reprodutiva, na qualidade de inovações tecnológicas assistenciais de integração de serviços, revela a ousadia e a adesão promissora dos gestores municipais às diretrizes da integralidade e da política de regionalização. Avaliadores precisam ser proativos nesse processo, resgatando claramente em seu trabalho a "visão ideal" e os princípios do Sistema Único de Saúde (SUS), a fim de influenciar o caminhar das reformas nessa direção, ainda que através de modelos experimentais e parciais de integração e/ou da regionalização preconizada na Norma Operacional de Assistência à Saúde (NOAS-SUS 01/2001). Nessa visão, a integração alinhar-se-ia ao conceito ampliado de integralidade como uma ação social que resulta da interação democrática entre os atores no cotidiano de suas práticas na oferta do cuidado de saúde, nos diferentes níveis de atenção do sistema 16 .

\section{Resumo}

Neste texto, partimos do pressuposto de que a integralidade da atenção é um eixo prioritário da investigação e avaliação dos serviços e sistemas de saúde, estruturados como redes assistenciais interorganizacionais que articulam dimensões clínicas, funcionais, normativas e sistêmicas em sua operacionalização, reconhecendo que nenhuma organização reúne a totalidade dos recursos e competências necessárias para a solução dos problemas de saúde de uma população, em seus diversos ciclos de vida. Em virtude da complexidade desse "sistema sem muros", que elimina as barreiras de acesso entre os diversos níveis de atenção, em resposta às necessidades de saúde nos âmbitos local e regional, julgamos oportuno compartilhar algumas "lições preliminares" aprendidas em experiências pessoais e na literatura sobre a integração de serviços, que nos parecem de interesse comum aos pesquisadores $e$ gestores comprometidos com a sua implantação.

Serviços de Saúde; Avaliação dos Serviços; Interação de Sistemas

\section{Colaboradores}

Z. Hartz colaborou na contextualização e redação do texto. A.-P. Contandriopoulos contribuiu com a teorização e discussão temática. 


\section{Referências}

1. Leatt P, Pink G, Guerriere M. Towards a Canadian model of integrated healthcare. Healthc Pap 2000; 1:13-55.

2. Giovanella L, Lobato L, Carvalho AL, Connil EM, Cunha EM. Sistemas municipais de saúde e a diretriz da integralidade da atenção: critérios para avaliação. Saúde Debate 2002; 26:37-61.

3. Contandriopoulos A-P, Denis J-L, Touati N, Rodriguez R. Intégration des soins: dimensions et mise-en-oeuvre. Ruptures 2001; 8:38-52.

4. Salhani D, Shea M, Lewko JH. Issues in the measurement of integrated service delivery in human services. Canadian Journal of Program Evaluation 1994; 9:1-14.

5. Fleury MJ, Denis JL. Proposition d'un modèle d'évaluation de la mise-en-oeuvre et des effets de la planification des programmes régionaux d'organisation des services de santé mentale au Québec. Canadian Journal of Program Evaluation 2000; 15:117-47.

6. Touati N, Contandriopoulos AP. Una expérience d'intégration des soins dans une zone rurale: les enjeux de la mise-en-oeuvre. Ruptures 2001; 8:93108.

7. Baubeau D, Marrel P. Les schémas régionaux d'organisation sanitaire et réseaux de soins. Prévenir les Risques du Métier 1994; 27:45-52.

8. Baubeau D, Marrel P. Planification et évaluation de l'organisation sanitaire. Apport des réseaux. Santé Publique 1995; 7:209-15.

9. Agence Nationale d' Accreditation et d'Évaluation en Santé. Principes d' évaluation des reséaux de santé. Paris: Agence Nationale d' Accreditation et d'Évaluation en Santé; 1999.
10. Centre d'Étude et de documentation en Economie de la Santé. L' évaluation des réseaux de soins. Bulletin d'Information en Écomomie de la Santé 2001; 37:1-4.

11. Brindis C, Hughes DC, Halfon N, Newacheck PW. The use of formative evaluation toassess integrated services for children. The Robert Wood Johnson Foundation Child Health Initiative. Eval Health Prof 1998; 21:66-90.

12. Hartz Z, Vieira-da-Silva L. Avaliação em saúde: dos modelos conceituais à prática na avaliação de programas e sistemas de saúde. Rio de Janeiro: Editora Fiocruz; no prelo.

13. Brown ADG, Michael J, Hicks N, Rourke JT, McMurtry RY, Brown JD. Hospitalization for ambulatory care-sensitive conditions: a method for comparative access and quality studies using routinely collected statistics. Can J Public Health 2001; 155-9.

14. Campbell MK. Assessment of regionalized perinatal programs. J Dev Phsysiol 1991; 15:125-31.

15. Contandriopoulos A-P, Champagne F, Denis JL, Avargues MC. L'évaluation dans le domaine de la santé: concepts et mpéthodes. Rev Epidemiol Santé Publique 2000; 48:517-39.

16. Pinheiro R. Integralidade e práticas de saúde: transformação e inovação na incorporação e desenvolvimento de novas tecnologias assistenciais de atenção aos usuários no SUS. Boletim ABEM 2003; 31:8-11.

Recebido em 26/Jan/2004 Aprovado em 18/Mai/2004 